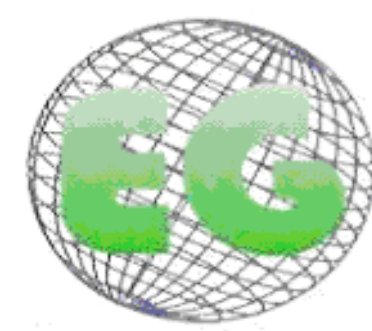

ISSN 1695-6141

$N^{\circ} 23$
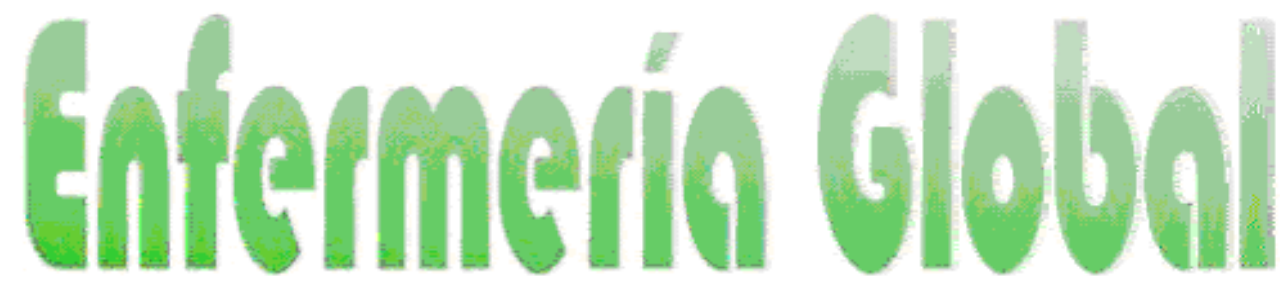

Revista electrónica trimestral de Enfermería

Julio 2011

www.um.es/eglobal/

\title{
¿Qué percepción tienen los pacientes de hematología sobre la higiene de manos - uso de guantes y enfermería?
}

Perception of haematology patients on hand hygiene - use of gloves and nursing

\author{
"Calero Romero, MR., **Llanes Correa, L. \\ *Enfermera. Hospital Universitario Virgen del Rocío. Sevilla. ${ }^{* *}$ Enfermera. Hospital General Juan Ramón \\ Jiménez. Huelva.
}

Palabras clave: Paciente hematológico; Aislamiento inverso; Higiene de manos; Infección; Seguridad del paciente.

Keywords: Haematology patient; Inverse isolation; Hand hygiene; Infection; Patient safety

\section{RESUMEN}

Objetivo: Conocer la opinión de los pacientes sobre la higiene de manos y el uso de guantes de los profesionales de enfermería que trabajan en la planta de hematología.

Diseño: Estudio cualitativo descriptivo mediante la técnica de grupo focal.

Población de Estudio: 5 pacientes de la unidad de hematología-oncología del Hospital Juan Ramón Jiménez de Huelva para poder realizar el grupo focal.

Técnica de recogida de datos: La técnica que se utilizó fue el grupo focal, se elaboró un guión para seguir un orden en la recogida de información.

Análisis de los datos: La transcripción del grupo focal fue literal.

Resultados: Todos los pacientes han observado que los enfermeros utilizan alhajas. Sólo uno de ellos ha observado el cuidado de las uñas de los enfermeros. Todos aseguran que utilizan las soluciones alcohólicas colocadas en las habitaciones, así como ninguno nunca llamó la atención a los profesionales por incumplimiento de alguna medida de higiene. Los sentimientos que pudiera provocar el incumplimiento de alguna medida es variado: "inseguridad, rabia"...entre otras.

Conclusiones: La higiene de manos y uso de guantes de los profesionales de enfermería es adecuada según sus pacientes, ya que nunca llamaron la atención por incumplimiento de alguna medida, estos utilizan guantes en sus técnicas y continuamente el lavado de manos con soluciones alcohólicas, aún así se debería de ofrecer información sobre el uso de alhajas y cuidado de uñas ya que no todos están de acuerdo que puedan producir infecciones, y también forman parte de la higiene de manos. 


\section{ABSTRACT}

Aim: To ascertain the opinion of patients about hand hygiene and the use of gloves by nursing professional working in the haematology ward.

Design: Qualitative, descriptive study using the focus group approach.

Study Population: 5 patients from the haematology-oncology ward of the Juan Ramón Jiménez Hospital of Huelva.

Data gathering technique: Focus group. A script was developed on the order of the collection of information.

Data analysis: Literal transcription of focus group.

Results: All patients observed that the nurses wore jewellery. Only one noticed that nurses looked after their fingernails. All stated that the alcohol solutions in the rooms were used and that no professional was advised of any non compliance with hygiene measures. Emotions that might cause non compliance of a measure were varied - "insecurity", "anger", amongst others.

Conclusions: Hand hygiene and use of gloves by nursing professionals is, according to the patients, correct, since professionals were never advised of non compliance with measures. They used gloves and continuously washed their hands in alcoholic solutions. Nevertheless, the wearing of jewellery and care of fingernails requires, ore information since there is no common agreement as to whether they produce infections, while they do form part of hand hygiene.

\section{INTRODUCCIÓN}

La calidad, como atributo esencial de los servicios prestados, es el elemento vertebrador del sistema sanitario. Las acciones diseñadas para su mejora continua, pretenden abarcar todas sus facetas. Asumir las necesidades y expectativas de ciudadanos y ciudadanas implica la identificación de su papel central y la obligación de establecer un sistema de garantías que lo preserve. ${ }^{1}$

La seguridad de los pacientes goza de la máxima prioridad entre las preocupaciones del sistema de salud, actividad cada vez más compleja, que entraña riesgos potenciales y en la que no existe un sistema capaz de garantizar la ausencia de eventos adversos, ya que se trata de una actividad en la que se combinan factores inherentes al sistema con actuaciones humanas. ${ }^{2}$

En este ámbito, la OMS, tomó como iniciativa lanzar la "Alianza Mundial para la Seguridad del Paciente", donde el tema elegido para el primer "Reto Mundial por la Seguridad del Paciente" (2005-2006), fue el de las infecciones asociadas a la atención sanitaria (IAAS), bajo el lema - Una atención limpia es una atención más segura -, y tiene como elemento clave, el fomento de la higiene de las manos para prevenir la transmisión de patógenos.

La higiene de las manos, una acción muy sencilla, sigue siendo la medida fundamental para reducir las infecciones asociadas a la atención sanitaria y la propagación de la resistencia a los antimicrobianos, pero algo compleja a la hora de poder evaluar por parte del sistema si se lleva o no a cabo, debido a que el factor de decisión humana, querer o no querer, juega un papel importante.

Por este motivo y debido a que en la revisión bibliográfica consultada no encontramos ningún estudio referente a la opinión de los pacientes sobre la higiene de manos y uso de guantes de los profesionales, se pensó que la mejor manera de ver la importancia que tiene esta acción para los pacientes y su seguridad frente a las IAAS, es hacer llegar al profesional sanitario, y más concretamente a enfermería, la percepción que tienen estos sobre el tema, ya que debido a los efectos de los tratamientos de quimioterapia pasan largas 
estancias en el hospital inmunodeprimidos, y por tanto son pacientes que están muy sensibilizados con el tema de la higiene y el lavado de manos, así como con las precauciones necesarias que se tienen que llevar a cabo a la hora de prestarle cuidados. Esta opinión nos podrá servir como herramienta, tanto para evaluar si se cumple o no dicha estrategia en seguridad del paciente como para sensibilizar aún más a los profesionales con el tema.

Así que el objetivo de este estudio fue: Conocer la opinión de los pacientes sobre la higiene de manos y el uso de guantes de los profesionales de enfermería que trabajan en la planta de hematología.

En los últimos años, las consecuencias sanitarias, sociales y económicas de los errores en la asistencia han conducido a organismos internacionales a reflexionar sobre la seguridad del paciente y la gestión de riesgos sanitarios y a desarrollar recomendaciones acerca de la prevención de los efectos adversos en la atención sanitaria y la formulación de estrategias en este ámbito, tal como la Alianza Mundial para la Seguridad del Paciente que promueve la Organización Mundial de la Salud. Enfatizando que las estrategias de seguridad y sus sistemas deben incluir acciones dirigidas a la prevención, detección y mitigación de los efectos adversos cada vez que ocurren, así como al análisis de sus causas, aprendizaje de los errores y la difusión de las lecciones aprendidas. ${ }^{3}$

La carga de morbilidad atribuible a las infecciones asociadas a la atención sanitaria es enorme: en el mundo, millones de pacientes resultan afectados cada año. Estas infecciones causan muertes y discapacidades, y propician la resistencia a los antibióticos. ${ }^{4}$

- En todo momento, hay en el mundo más de 1,4 millones de personas que padecen infecciones contraídas en centros sanitarios.

- Entre el $5 \%$ y el $10 \%$ de los pacientes ingresados en hospitales del mundo desarrollado contraen una o más infecciones. ${ }^{5}$

El lavado de manos es la principal medida para evitar las infecciones nosocomiales de origen exógeno. Así, en el año 1846, Semmelweis fue el primero que relacionó el lavado de manos con la transmisión de enfermedades infecciosas. Desde este momento fueron muchos los que trataron este tema, pero es Eickoff en 1980 el que establece los niveles de eficacia de las medidas de control de la infección nosocomial y dice: el lavado de manos constituye una de las medidas de eficacia probada para evitar la contaminación o infección.

El compromiso a nivel nacional de priorizar la prevención de estas infecciones puede mejorar enormemente la seguridad del paciente.

La extensión de estas medidas de mejora seguridad (bioseguridad-higiene de manos) por parte del sistema sanitario en todas las comunidades autónomas, tienen como estrategia global: 6

$\checkmark$ La facilitación de los recursos necesarios para la mejora de la higiene de manos, como pueden ser la soluciones alcohólicas instaladas en la puerta de las habitaciones, control de enfermería...

$\checkmark$ La información y sensibilización tanto de los profesionales como de los propios pacientes sobre el tema, a través de folletos informativos, carteles, pósters....

$\checkmark$ La formación de los profesionales en seguridad. 
$\checkmark$ La evaluación de las intervenciones y estrategias establecidas y llevadas a cabo, así como la prevalencia en este caso de IAAS. ${ }^{7}$

\section{MATERIAL Y MÉTODOS}

El abordaje de este estudio se realizó siguiendo una metodología cualitativa, usando una técnica de grupo focal, por ser fácil de usar, aportar información rápida, permitir la interacción del grupo, así como estimular la generación de ideas de forma simultánea.

El ámbito de estudio fue la planta de Hematología-Oncología del Hospital General Juan Ramón Jiménez de Huelva.

La población estudiada fueron 5 pacientes seleccionados de dicha planta, con los que se realizó la técnica de grupo focal y cuyo criterio de inclusión, debido a que la planta es de las especialidades de hematología y oncología, fue el siguiente: ser paciente de hematología, haber estado ingresado durante el mes de Febrero de 2010 completo y además en aislamiento inverso, por lo que nos quedó una población total de 5 pacientes.

Posteriormente, se les explicó al grupo de pacientes el fin del estudio y si aceptaban a participar, al no negarse ninguno se concretó un día para realizar la técnica de grupo focal. Se realizó una sesión de unos 20 minutos aproximadamente, debido a la situación inestable de dichos pacientes.

En la sala que se les citó había una mesa redonda donde se las asignó un sillón a cada uno, en ella había un modelador del grupo y un anotador para anotar todo aquello que resaltaba. Tras explicar en profundidad la finalidad del estudio y pedir permiso, se comenzó a grabar, siguiendo el guión elaborado para dicha sesión, que se muestra a continuación:

1. ¿Habéis observado si el profesional de enfermería utiliza anillos, pulseras, relojes...a la hora de prestar sus cuidados?

2. ¿Pensáis que el uso de alhajas puede ser causante de infección?

3. ¿Ha observado si el profesional de enfermería mantiene el cuidado de las uñas (onicofagia)?

4. ¿Utiliza el personal de enfermería la solución alcohólica instalada en la puerta de habitaciones antes y después de entrar a la habitación?

5. ¿Utiliza el profesional de enfermería guantes cuando realiza alguna técnica?

6. ¿Qué sentimiento despierta cuando observáis que el profesional de enfermería no cumple con alguna de las medidas comentadas antes?

7. ¿Habéis llamado la atención a algún profesional de enfermería por algún incumplimiento de lo comentado anteriormente?

8. ¿Pensáis que la higiene de manos es una medida importante para prevenir las infecciones?

Para el análisis de los datos se realizó una transcripción literal de los resultados, en un primer lugar se hizo una lectura generalizada de la sesión para resumir la información.

\section{RESULTADOS}

Se recogieron opiniones de un total de 5 pacientes ingresados en la planta de hematologíaOncología del Hospital Juan Ramón Jiménez. 
En cuanto a la utilización de alhajas de los profesionales cuando prestan sus cuidados, todos los pacientes coinciden que han observado que todos los enfermeros utilizan alguna alhaja : "los enfermeros de esta planta utilizan todos algún que otro adorno", "las enfermeras mujeres llevan todas anillos y, en general, relojes, soy muy observadora, la verdad que me suelo fijar en todo, con todo el tiempo que tienes aquí, te das cuenta de todo ", "los enfermeros utilizan por lo general alguna alhaja", " los enfermeros que trabajan en esta planta utilizan alhajas, relojes, pulseras, anillos", " los profesionales que trabajan aquí utilizan alguna de las cosas que han nombrado mis compañeros, como cualquier otra persona".

A lo que se refiere si el uso de dichas alhajas puede causar algún tipo de infección, tres de los cinco pacientes están de acuerdo en que pueden transmitir infección y dos no: " el uso de pulseras, anillos y todas esas cosas pueden producir infecciones, están con la persona siempre en la calle, en casa, en el hospital, y por mucho que te laves las manos, no creo que se queden libres de bichitos del ambiente", " pienso que sí puede producir infecciones por todo lo que ha dicho mi compañero, y además los enfermeros están en contacto con todos los pacientes, entrando y saliendo, sacando sangre, curando, puede pasarse infecciones de unos a otros", " estoy de acuerdo con lo que se ha dicho, al trabajo hay que venir libre de todas esas cosas, una enfermera tiene un reloj colgado en el bolsillo, sería buena opción”, pienso que no tienen por qué producir infecciones la utilización de esas cosas, porque la enfermera estoy segura porque aquí hay muy buenos profesionales de que se lavan las manos y después vemos que se ponen guantes", " estoy de acuerdo con mi compañera, si utilizas anillos, pulseras... se pueden tapar con los guantes limpios y no pasa nada".

En lo que se refiere al cuidado de las uñas de los enfermeros, solo un paciente ha observado eso, los demás coinciden en que nunca observaron las uñas: "sí, todos los profesionales, bueno con los que me han tratado, tienen sus uñas muy bien cuidadas, limpias, cortadas", "nunca me he fijado en las uñas, será porque la mayoría de las veces los enfermeros tienen los guantes puestos", el resto de pacientes está de acuerdo con este último.

Con respecto a la utilización de la solución alcohólica antes y después de entrar a la habitación, todos los pacientes coinciden que se utiliza, excepto uno: "sí, están continuamente echándose el alcohol ese en las manos", "sí se utiliza, aunque creo que más al salir que al entrar en las habitaciones", "se echan continuamente en las manos, aunque no estén sacando sangre y tomando tensiones, ya como costumbre, aunque sea para hablar con los pacientes", " creo que se utiliza más al entrar", " pues todos los enfermeros no utilizan al entrar y salir en las habitaciones".

En lo que se refiere a la utilización de guantes en la realización de técnicas, todos los pacientes coinciden en que se utilizan los guantes: "sí, siempre que van a hacer algo vienen con los guantes, a veces hay enfermeros que tienen guantes hasta para hablarte, porque los llevan siempre puestos, hay otros que a veces no llevan los guantes para hablar, para tomarte la tensión“, " sí, siempre que van a hacerte alguna cosa, vienen preparados", el resto afirma lo comentado.

Con respecto al sentimiento que despierta el incumplimiento de alguna medida de las comentadas anteriormente, los pacientes argumentan lo siguiente : "no tiene que despertar sentimiento, porque esas normas están obligados a cumplirlas los enfermeros", " a mí me da rabia", " pues si te soy sincera, me transmite inseguridad, no me siento segura de que esa persona me trate en ese momento", " me quedo observando y guardo silencio, no me puedo meter en el trabajo de nadie, ellos son los profesionales y estarán informados de lo que 
puede pasar", " no estoy de acuerdo con mi compañero, yo como paciente tengo derecho a mirar por mí, y mucho más si ellos no lo hacen, las cosas hay que decirlas, a veces hay mucho trabajo y puede ser despiste, pero se le dice, y no creo que pase nada, no he visto que se incumpla alguna medida en esta unidad, son todos muy buenos profesionales", todos están de acuerdo con el paciente cinco.

En lo que se refiere a si han llamado la atención a algún enfermero por algún incumplimiento, todos coinciden en que no ha sido necesario en esta planta: "son todos muy profesionales", el resto afirma que están de acuerdo con su compañero.

Con respecto a lo último comentado, sobre si la higiene de manos es una medida importante para prevenir infecciones, todos coinciden en que es una medida muy importante para prevenir cualquier tipo de infección " es una medida que aprendemos de pequeños", " quién de pequeño no le han hecho lavarse las manos a cada instante cuando llegabas de jugar, del colegio e ibas a comer, niño que te pones malo","sí, es algo que se aprende desde pequeño, es verdad", " sí es una de las medidas que los padres desde chico están encima de ti al igual que el lavado de dientes", "sí es importante la higiene de manos, con las manos tocamos todo".

\section{DISCUSIÓN Y CONCLUSIONES}

Los resultados de este estudio están referidos a conocer la opinión de los pacientes sobre la higiene de manos y uso de guantes de los profesionales de enfermería que le prestan cuidados.

En la revisión bibliográfica consultada no se ha encontrado ningún estudio referente a la opinión de los pacientes con respecto a la higiene de manos y uso de guantes.

De los resultados del grupo focal destaca que por lo que respecta a la utilización de alhajas de los profesionales todos los pacientes han observado que los enfermeros de la planta de hematología-oncología utilizan alhajas, también coinciden que todos los enfermeros utilizan guantes a la hora de realizar alguna técnica, así como que nunca han tenido que llamar la atención a ningún profesional por el incumplimiento de alguna medida comentada a lo largo de la discusión, todos ellos piensan que la higiene de manos es muy importante para prevenir las infecciones, destaca que todos lo saben bien desde pequeños.

Con respecto a los sentimientos que despierta el incumplimiento de alguna medida las repuestas son muy variadas, pero para ninguno nada positivo, en esta unidad no han visto el incumplimiento.

En el resto de las preguntas que se debatieron no todos están de acuerdo, dos de los cinco pacientes no creen que el uso de dichas alhajas pueda ser causante de infección, solo uno de ellos observa que las uñas de los profesionales de enfermería están bien cuidadas, así como solo uno de los cinco refiere observar que no todos los profesionales de enfermería hacen uso de las soluciones alcohólicas instaladas a la entrada de las habitación de la planta.

Los pacientes crean lazos afectivos con los profesionales de la planta de hematologíaoncología, por sus largas estancias y diversas, evaluando positivamente lo analizado en el grupo focal, podría ser que estos pacientes evalúan la globalidad de los cuidados, sin entrar específicamente en detalles, como es en este caso la higiene de manos y uso de guantes, 
ya que estos se encuentran muy satisfechos con los cuidados que reciben por parte de los profesionales.

\section{CONCLUSIONES}

La higiene de manos y uso de guantes de los profesionales de enfermería es adecuada, ya que los pacientes argumentan que los profesionales de enfermería utilizan guantes, utilizan generalmente las soluciones alcohólicas y nunca han tenido que llamar la atención a los profesionales por incumplimiento de alguna medida de lo comentado.

Todos comentan que la higiene de manos es una medida muy importante para prevenir infecciones, pero no incluyen dentro de esa higiene de manos, el uso de alhajas ni el cuidado de las uñas (onicofagia). Los pacientes saben que los profesionales llevan alhajas a la hora de realizar alguna técnica, y la mayoría nunca observó el cuidado de las uñas. Las opiniones son variadas, no todos están de acuerdo que el uso de las alhajas pueda producir también infecciones y por tanto, al no darle importancia a esas medidas nunca han llamado la atención a los profesionales.

Sería deseable ofrecer información sobre otras medidas higiénicas que también entran a formar parte en la higiene de manos, como son el uso de las alhajas y el cuidado de las uñas de los profesionales.

\section{REFERENCIAS BIBLIOGRÁFICAS}

1 Plan de Calidad para el Sistema Nacional de Salud. Módulo de Formación Proyecto Bacteriemia cero. Ministerio de Sanidad y Consumo. Madrid. 2008

2 Estrategia para la seguridad del paciente. Consejería de Salud. Junta de Andalucía. Sevilla.2006

${ }^{3}$ Alianza Mundial para la Seguridad del paciente. Directrices de la OMS sobre la higiene de manos en la atención sanitaria. OMS. 2005 http://www.who.int/patientsafety/challenge/en/

${ }^{4} \mathrm{Pi}$-Sunyer, T. Experiencia sobre el lavado de manos en una institución sanitaria. IMAS, Instituto Municipal de Asistencia Sanitaria. Barcelona.2006

${ }^{5}$ Alianza Mundial para la Seguridad del paciente. Primer Reto Mundial para la Seguridad del paciente: una atención limpia es una atención más segura. OMS 2005-2006.

6 Estrategia en seguridad del paciente. Planificación Sanitaria. Calidad y Evaluación Sanitaria. Ministerio de Sanidad y Consumo. Recomendaciones del Taller de Expertos celebrado en febrero. Madrid. 2005.

${ }^{7}$ Vázquez $P$, Lebrero R, Fernández E, Fernández $M^{a}{ }^{a} D$, Irastorza $M^{a}{ }^{a}$, Limón J. Estrategia de implementación de la práctica segura de higiene de manos en Atención Primaria en Andalucía. Servicio Andaluz de Salud. Sevilla. 2008.

ISSN 1695-6141

@) COPYRIGHT Servicio de Publicaciones - Universidad de Murcia 